\title{
Adaptive Context for a Discrete Universal Denoiser
}

\author{
Georgy Gimel'farb \\ Department of Computer Science, Tamaki Campus \\ The University of Auckland, Auckland, New Zealand \\ g.gimelfarb@auckland.ac.nz \\ http: //www.tcs.auckland.ac.nz/ georgy
}

\begin{abstract}
Statistical analysis of spatially uniform signal contexts allows Discrete Universal Denoiser (DUDE) to effectively correct signal errors caused by a discrete symmetric memoryless transmission channel. The analysis sets no limits on a probability signal model apart from stationarity and ergodicity. Statistics of signal contexts are used first to learn the probability of errors and then to detect and correct the errors. Therefore a proper choice of context is an essential prerequisite to the practical use of DUDE. We propose to use the maximum likelihood estimate of context assuming the signals are modelled with a nonparametric generic Markov-Gibbs random chain or field. The model adds to stationarity and ergodicity only one more condition, namely, pairwise dependences between each signal and its context. Experiments with noisy binary images confirm a feasibility of such adaptive context, show some advantages of DUDE over more conventional median filtering, and relate the choice of a proper context size to the maximum entropy of the context statistics used for image denoising.
\end{abstract}

\section{Introduction}

Although denoising is one of the most extensively studied areas of signal and image processing, the variety of models and techniques involved is permanently growing (see, e.g., $[1-3,5]$ to cite a few). Discrete Universal Denoiser (DUDE) proposed recently in [6] recovers an original $1 \mathrm{D}$ sequence or 2D array of signals by analysing signal contexts in a noisy signal set corrupted by a memoryless symmetric transmission channel. In spite of simplicity, such signal model is of interest in many important practical applications where probability characteristics of signals are unknown, except for stationarity and ergodicity. The context consists of signals in a fixed (translation invariant) neighbourhood of each position in the sequence or array. Because marginal probability distributions of stationary and ergodic signals are translation invariant, DUDE uses relative frequencies of different signals with the same context first to learn the error probability for the channel and then to detect and correct errors.

Efficiency of DUDE essentially depends on context geometry, i.e. the number and relative positions of neighbours. Generally, most adequate geometry depends on signal sets to denoise. But typically the context is pre-defined by heuristic considerations, e.g., signals in a fixed rectangular window around each position. This paper attempts to directly estimate the best context assuming that the noisy signals are samples of a nonparametric generic Markov-Gibbs random chain or field [4]. This spatially uniform 
model is also stationary and ergodic, but it adds one more general restriction, namely, only pairwise dependencies between each signal and its context. This restriction allows for the approximate maximum likelihood estimate (MLE) of the context.

The paper is organised as follows. Section 2 describes DUDE in brief, compares it to a conventional median filter denoiser (MFDE), and discusses the MLE of context for the Markov-Gibbs signal model. Experiments with DUDE and MFDE and an entropybased choice of the context size are presented in Section 3.

\section{Maximum Likelihood Context}

For brevity, we address only 2D binary images, although with obvious changes our consideration applies also to $2 \mathrm{D}$ arrays and $1 \mathrm{D}$ sequences of multi-level signals. Let $\mathbf{R}=\{(x, y): x=0, \ldots, X-1 ; y=0, \ldots, Y-1\}$ denote an arithmetic 2D lattice supporting signal arrays $g: \mathbf{R} \rightarrow \mathbf{Q}$ where $\mathbf{Q}=\{0, \ldots, Q-1\}$ is a finite set of scalar signals $(Q=2$ for binary images). A translation invariant neighbourhood of size $K$ for each site $(x, y) \in \mathbf{R}$ effects conditional probabilities of signal values $g(x, y) \in \mathbf{Q}$. Its geometry is specified by a set of $(x, y)$-increments $\mathbf{N}=\left\{\left(\xi_{k}, \eta_{k}\right): k=1, \ldots, K\right\}$ such that each signal $g(x, y)$ has the context $\mathbf{C}_{j}=\{g(x+\xi, y+\eta):(\xi, \eta) \in \mathbf{N} ;(x+\xi, y+$ $\eta) \in \mathbf{R}\}$. For binary images, there are $2^{K}$ different contexts $\mathbf{C}_{j} ; j \in\left\{0, \ldots, 2^{K}-1\right\}$, such that $j$ is the binary number $q_{1} q_{2} \ldots q_{K}$ where $q_{k} \in \mathbf{Q}$.

Both DUDE and MFDE use contexts to decide whether a binary signal is true or corrupted. The passive MFDE follows the majority rule: each signal $g(x, y)$ is true if at least half of the context signals $(g(x+\xi, y+\eta):(\xi, \eta) \in \mathbf{N})$ have the same value. The active DUDE finds $2^{K+1}$ conditional frequencies $f\left(q \mid \mathbf{C}_{j}\right) ; q \in \mathbf{Q}$, of signals in a pixel, given its context $\mathbf{C}_{j}$ of size $K ; f\left(0 \mid \mathbf{C}_{j}\right)+f\left(1 \mid \mathbf{C}_{j}\right)=1$. If the minimum of these two frequencies is less than a certain threshold, $\theta$, then such "less frequent" signal with this particular context is assumed to be noisy and will be reversed. The threshold derived in [6] depends on the noise probability estimated by the minimum conditional frequency of signals over all the contexts: $\operatorname{Pr}_{\text {noise }} \cong \min _{j, q}\left\{f\left(q \mid \mathbf{C}_{j}\right\}\right.$.

We consider a noisy signal array $\mathbf{g}=(g(x, y):(x, y) \in \mathbf{R})$ as a sample of a Markov-Gibbs random field with translation invariant geometric structure of pairwise dependencies between signals [4]. The model is specified with the Gibbs probability distribution

$$
\operatorname{Pr}(\mathbf{g} \mid \mathbf{N}) \propto \exp \left(\sum_{(x, y) \in \mathbf{R}}\left(V(g(x, y))+\sum_{(\xi, \eta) \in \mathbf{N}} V_{\xi, \eta}(g(x, y), g(x+\xi, y+\eta))\right)\right)
$$

Here, $V: \mathbf{Q} \rightarrow \mathbf{U}$ and $V_{\xi, \eta}: \mathbf{Q} \rightarrow \mathbf{U}$ are Gibbs potentials and $\mathbf{U}$ is the set of real numbers bounded from above. Both the potentials for and spatial geometry $\mathbf{N}$ of interdependent signal pairs are not predefined, may differ for different types of signals, and are estimated from a given signal array $\mathbf{g}$.

The $\log$-likelihood $L(\mathbf{N} \mid \mathbf{g})=\frac{1}{X Y} \log \operatorname{Pr}(\mathbf{g} \mid \mathbf{N})$ maximised by the potentials and depending only on the neighbourhood $\mathbf{N}$ is analytically approximated as $L(\mathbf{N} \mid \mathbf{g})=$ $-\sum_{q=0}^{Q-1} f(q \mid \mathbf{g}) \log f(q \mid \mathbf{g})+\sum_{(\xi, \eta) \in \mathbf{N}} d_{\xi, \eta}(\mathbf{g})$ by adapting the derivation in [4] to different marginal signal probabilities. Here, $d_{\xi, \eta}(\mathbf{g})$ is the squared distance between 

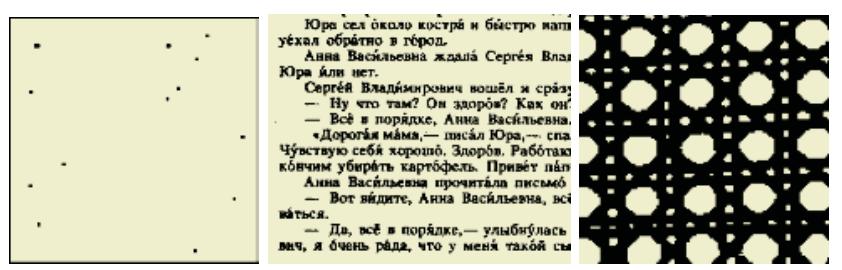

Fig. 1. Noiseless "Stars", "Cyrillic Text”, and "Brodatz's Cane".
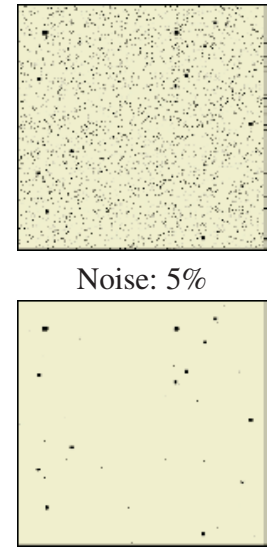

$e_{\text {DUDE }}=0.06 \%$

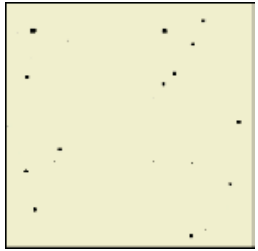

$e_{\mathrm{MFDE}}=0.04 \%$

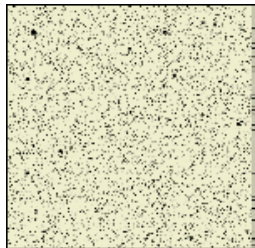

$10 \%$

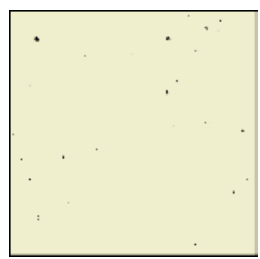

$0.2 \%$

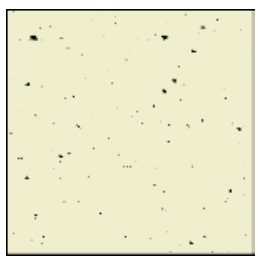

$0.4 \%$

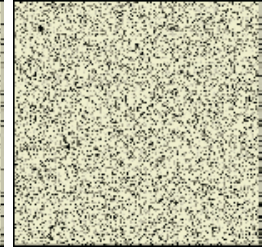

$20 \%$

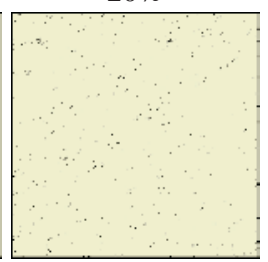

$1.1 \%$

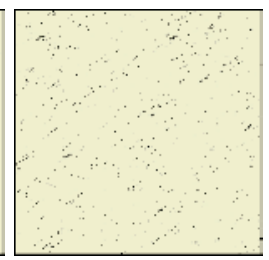

$1.5 \%$

Fig. 2. Noisy and denoised "Stars".

Table 1. Estimates of the noise probability $\operatorname{Pr}_{\text {noise }}$ in DUDE.

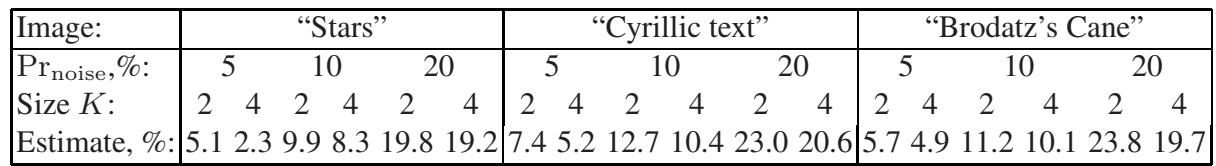

the frequency distribution $\mathbf{F}_{\xi, \eta}(\mathbf{g})=\left\{f_{\xi, \eta}(q, s \mid \mathbf{g}): q, s \in \mathbf{Q}^{2}\right\}$ of actual signal cooccurrences $(g(x, y)=q, q(x+x i, y+\eta)=s)$ in translation invariant pixel pairs $(x, y),(x+\xi, y+\eta):(x, y) \in \mathbf{R} ;(x+\xi, y+\eta) \in \mathbf{R}$ and the like distribution of independent signals with the same marginal probabilities $f(q \mid \mathbf{g}) ; q \in \mathbf{Q}$ : $d_{\xi, \eta}(\mathbf{g})=\sum_{(q, s) \in \mathbf{Q}}\left(f_{\xi, \eta}(q, s \mid \mathbf{g})-f(q \mid \mathbf{g}) f(s \mid \mathbf{g})\right)^{2}$. Therefore, the approximate MLE 


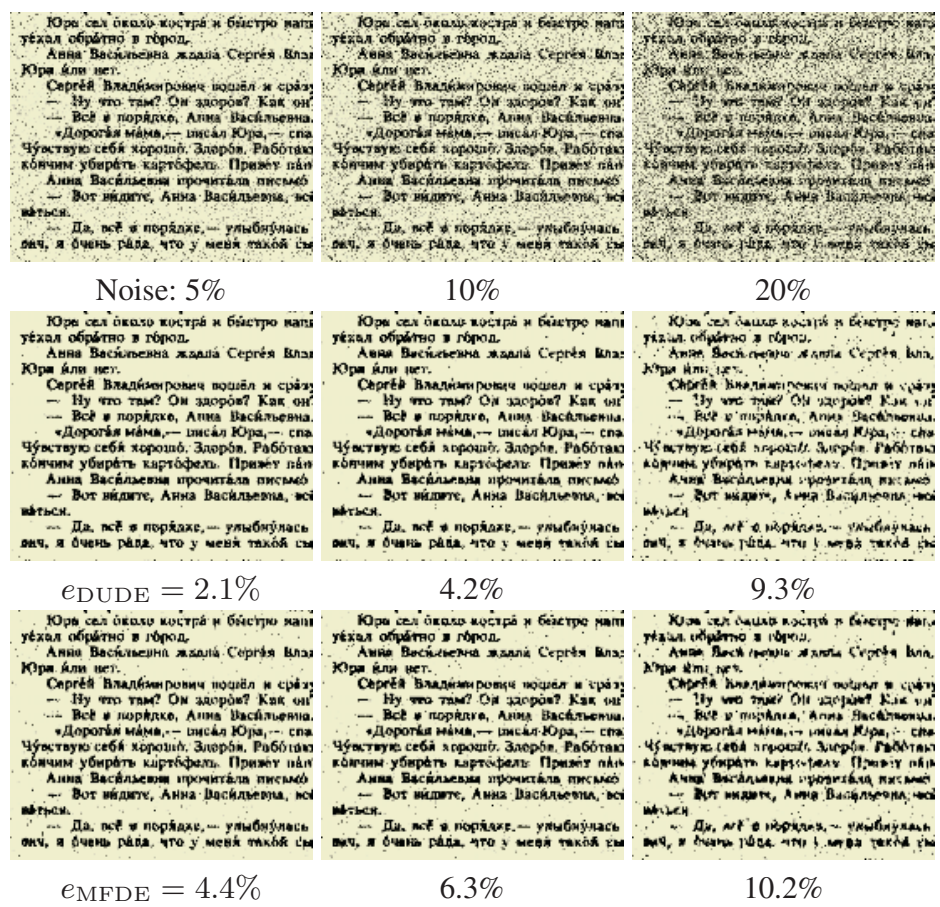

Fig. 3. Noisy and denoised "Cyrillic Text".

of the context of a given size $K$ is specified for a particular signal array $\mathrm{g}$ by the $K$ top-rank distances $d_{\xi, \eta}(\mathbf{g})$. But it is still necessary to quantitatively relate the context size $K$ ensuring better performance to the context statistics used by DUDE.

\section{Adaptive Maximum Entropy Contexts}

Figure 1 shows initial binary images "Stars", "Cyrillic text", and "Brodatz's Cane" used in experiments and having $0.3 \%, 21 \%$, and $66 \%$ of black pixels, respectively. The noisy versions obtained by modelling a symmetric transmission channel are presented in Figs. 2-4 together with results of denoising. The channel makes independent random bit inversions with a fixed probability $p=0.05,0.10$, or 0.20 . Experiments below compare DUDE with the adaptively chosen contexts to MFDE with the same contexts.

For small context sizes, the minimum relative frequency of contexts is a reasonable probability estimate. But as mentioned in [6], it fails for larger contexts due to a large number of too rare or simply absent signal configurations in a given image. Thus we estimate the noise probability and the relevant threshold using only small radially symmetric contexts. Table 1 shows these latter estimates for the symmetric contexts of size 2 and 4 in the noisy images in Figs. 2-4. The estimate for size $K=4$ fails for "Stars" because of a very small black area. To conduct all experiments in the same conditions, the probability estimates for the smallest symmetric context of size 2 is used below. For two other images the estimates for the size 4 are more adequate, but changes in quality 


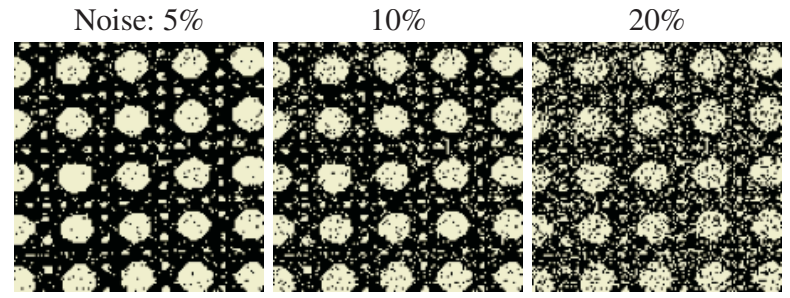

the best DUDE-context in the window $13 \times 13$

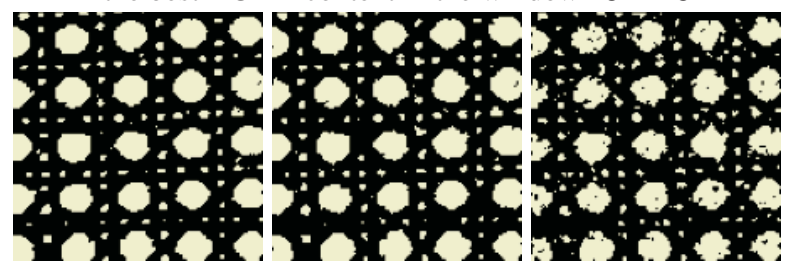

$e_{\text {DUDE }}=1.6 \%$

$2.7 \%$

$7.6 \%$

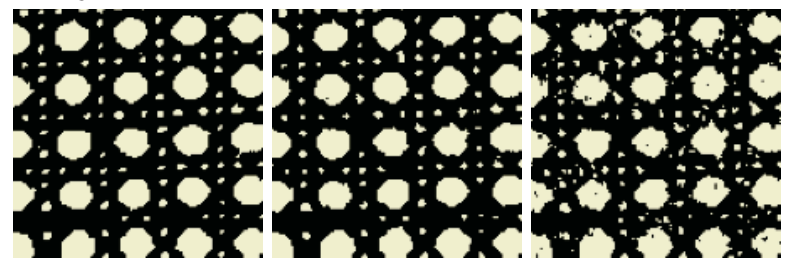

$e_{\mathrm{MFDE}}=2.2 \%$

$3.5 \%$

$7.1 \%$

the best DUDE-context in the window $81 \times 81$

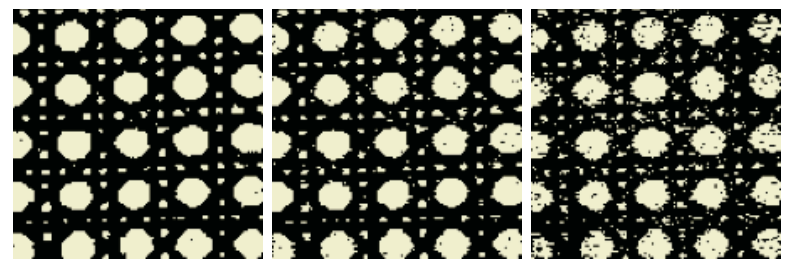

$e_{\mathrm{DUDE}}=1.2 \%$

$3.4 \%$

$9.4 \%$

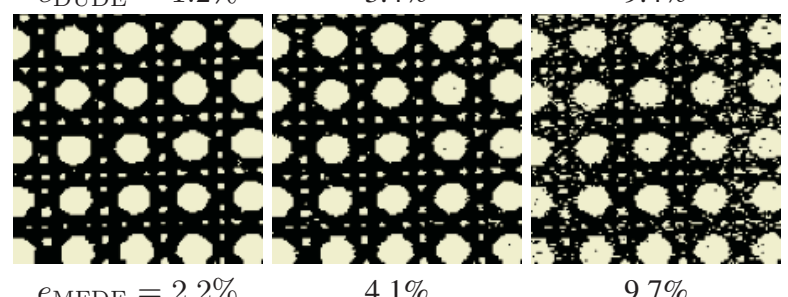

$e_{\mathrm{MFDE}}=2.2 \%$

$4.1 \%$

$9.7 \%$

Fig. 4. Noisy and denoised "Brodatz's Cane".

of denoising are marginal. In all our experiments asymmetric neighbourhoods ranked below symmetric ones. Thus, we use below only these latter.

Tables 2-4 present residual errors after image DUDE and MFDE using radially symmetric adaptive contexts $\mathbf{N}=\left\{\left(\xi_{i}, \eta_{i}\right),\left(-\xi_{i},-\eta_{i}\right): i=1, \ldots, K\right.$ of size $K=$ 
Table 2. Denoising of "Stars": $0.3 \%$ black (B) and $99.7 \%$ white $(\mathrm{W})$ pixels ( $e_{\ldots}$ denotes the total residual error after denoising; "B" and "W" indicate the individual residual errors for the black and white areas of the noiseless image, respectively; the maximum entropy and the minimum total errors are boldfaced; the individual errors are in italic and underlined).

\begin{tabular}{|c|c|c|c|c|c|c|c|c|c|c|}
\hline Half-size $i$ & 1 & 2 & 3 & 4 & 5 & 6 & 7 & 8 & 9 & 10 \\
\hline \multicolumn{11}{|c|}{ Noise: 5\% (actual: B 2.19\%; W 5.09\%); $\theta=0.096$} \\
\hline$\left(\xi_{i}, \eta_{i}\right)$ & $\overline{(0,1)}$ & $(1,0)$ & $(0,2)$ & $(1,1)$ & $\overline{(2,0)}$ & $(0,2)$ & $\overline{(1,-2)}$ & $(0,3)$ & $\overline{(1,-1)}$ & $(5,-1)$ \\
\hline Entropy & 1.0 & 2.2 & 2.5 & 2.2 & 1.6 & 1.3 & 1.2 & 1.0 & 0.9 & 0.8 \\
\hline$e_{\mathrm{DUDE}}, \%$ & 0.2 & 0.08 & 0.06 & 0.08 & 0.1 & 0.2 & 0.3 & 0.4 & 0.5 & 0.8 \\
\hline $\mathrm{B}, \%$ & 53.6 & 4.9 & 5.5 & 4.4 & 3.8 & 2.2 & 2.2 & 2.2 & 2.2 & 2.2 \\
\hline $\mathrm{W}, \%$ & 0.01 & 0.06 & $\underline{0.05}$ & 0.07 & 0.1 & 0.2 & 0.3 & 0.4 & 0.5 & 0.7 \\
\hline$e_{\mathrm{MFDE}}, \%$ & 0.8 & 0.1 & $\overline{0.04}$ & 0.05 & 0.05 & 0.1 & 0.1 & 0.1 & 0.1 & 0.2 \\
\hline $\mathrm{B}, \%$ & 2.7 & 2.2 & 3.3 & 14.2 & 15.3 & 30.1 & 38.3 & 50.3 & 48.1 & 64.5 \\
\hline $\mathrm{W}, \%$ & 0.1 & 0.03 & 0.01 & $\underline{0.0}$ & 0.0 & 0.0 & 0.0 & 0.0 & 0.0 & 0.0 \\
\hline \multicolumn{11}{|c|}{ Noise: $10 \%$ (actual: B 10.9\%; W 9.9\%); $\theta=0.178$} \\
\hline$\left(\xi_{i}, \eta_{i}\right)$ & $\overline{(0,1)}$ & $(1,1)$ & $(0,2)$ & $(2,0)$ & $\overline{(2,-1)}$ & $(1,0)$ & $(2,2)$ & $(1,-2)$ & $(1,2)$ & $(4,2)$ \\
\hline Entropy & 1.0 & 1.9 & 2.9 & 2.8 & 2.3 & 1.8 & 1.5 & 1.3 & 1.1 & 1.0 \\
\hline$e_{\mathrm{DUDE}}, \%$ & 0.3 & $\underline{0.2}$ & 0.2 & 0.2 & 0.4 & 0.5 & 0.9 & 1.3 & 1.9 & 2.5 \\
\hline $\mathrm{B}, \%$ & 99.5 & 65.6 & 61.2 & 42.6 & 27.9 & 12.0 & $\underline{10.9}$ & 10.9 & 10.9 & 10.9 \\
\hline $\mathrm{W}, \%$ & $\underline{0.0}$ & 0.03 & 0.05 & 0.1 & 0.3 & 0.5 & 0.9 & 1.3 & 1.8 & 2.5 \\
\hline$e_{\mathrm{MFDE}}, \%$ & 2.8 & 1.0 & 0.4 & 0.2 & 0.2 & 0.1 & 0.1 & 0.2 & 0.2 & 0.2 \\
\hline $\mathrm{B}, \%$ & $\underline{13.1}$ & 24.6 & 27.3 & 33.9 & 42.6 & 37.2 & 50.8 & 56.3 & 61.2 & 71.6 \\
\hline $\mathrm{W}, \%$ & 2.8 & 0.9 & 0.3 & 0.1 & 0.03 & 0.02 & $\underline{0.0}$ & 0.0 & 0.0 & 0.0 \\
\hline \multicolumn{11}{|c|}{ Noise: $20 \%$ (actual: B 21.9\%; W 19.9\%); $\theta=0.317$} \\
\hline$\left(\xi_{i}, \eta_{i}\right)$ & $\overline{(0,2)}$ & $\overline{(2,-2)}$ & $\overline{(3,-5)}$ & $(1,-1)$ & $(4,6)$ & $(1,1)$ & $(3,4)$ & $(6,3)$ & $(2,-1)$ & $(2,4)$ \\
\hline Entropy & 1.0 & 1.5 & 2.5 & 3.1 & 3.1 & 2.5 & 1.9 & 1.5 & 1.1 & 0.8 \\
\hline$e_{\mathrm{DUDE}}, \%$ & 0.3 & 0.3 & 0.3 & 0.5 & 1.1 & 2.3 & 4.5 & 7.4 & 10.2 & 13.1 \\
\hline $\mathrm{B}, \%$ & 99.5 & 99.5 & 99.5 & 92.8 & 80.3 & 64.5 & 51.4 & 43.7 & 32.2 & 28.4 \\
\hline $\mathrm{W}, \%$ & $\underline{0.0}$ & 0.0 & 0.02 & 0.2 & 0.4 & 2.1 & 4.3 & 7.3 & 10.1 & $\overline{13.0}$ \\
\hline MFDE,$\%$ & 10.0 & 6.0 & 3.5 & 2.2 & 1.5 & 1.0 & 0.7 & 0.6 & 0.5 & 0.4 \\
\hline $\mathrm{B}, \%$ & $\underline{32.8}$ & 50.8 & 69.9 & 67.8 & 78.1 & 75.4 & 82.0 & 88.5 & 87.4 & 92.4 \\
\hline $\mathrm{W}, \%$ & $\overline{10.2}$ & 5.9 & 3.3 & 2.0 & 1.2 & 0.8 & 0.5 & 0.3 & 0.2 & $\underline{0.1}$ \\
\hline
\end{tabular}

$2,4, \ldots, 20$. These results suggest that the entropy of distribution of the minimum relative frequencies of signal contexts roughly indicates the best choice of $K$. In the most cases, the maximum entropy either points directly to the context yielding the smallest residual error or to the adjacent variant. Apart from "Stars" under most intensive noise, DUDE always outperforms MFDE, but both the denoisers are opposite with respect to which areas are successfully denoised or additionally corrupted.

The channel with $5 \%$ noise had actually corrupted only $2.2 \%$ of the small black "Stars". The best DUDE result fot the context of size 3 is slightly worse than of MFDE with the same context. Both DUDE and MFDE additionally corrupt the black areas (to $5.5 \%$ and $3.3 \%$, respectively) while almost completely clean the white background $(0.05 \%$ and $0.01 \%$ of the residual noise, respectively, comparing to the initial $5.09 \%)$. When the context size increases, DUDE corrupts the black area less (down to 2.2\%) but simultaneously leaves a bit more noisy white area (up to $0.7 \%$ ). MFDU behaves in 
Table 3. Denoising of "Cyrillic text": $20.7 \%$ black (B) and $79.3 \%$ white (W) pixels (the same notation as in Table 2).

\begin{tabular}{|c|c|c|c|c|c|c|c|c|c|c|}
\hline Half-size $i$ & 1 & 2 & 3 & 4 & 5 & 6 & 7 & 8 & 9 & 10 \\
\hline \multicolumn{11}{|c|}{ Noise: 5\% (actual: B 4.98\%; W 5.06\%); $\theta=0.137$} \\
\hline$\left(\xi_{i}, \eta_{i}\right)$ & $(1,0)$ & $(0,1)$ & $(1,-1)$ & $(1,1)$ & $(2,0)$ & $(0,2)$ & $(0,5)$ & $(0,4)$ & $(2,1)$ & $(2,-1)$ \\
\hline Entropy & 1.0 & 2.4 & 3.3 & 3.7 & 3.5 & 2.6 & 1.7 & 1.1 & 0.7 & 0.5 \\
\hline$e_{\mathrm{DUDE}}, \%$ & 3.5 & 2.8 & 2.3 & 2.1 & 2.1 & 2.0 & 2.1 & 2.2 & 2.3 & 2.4 \\
\hline $\mathrm{B}, \%$ & 12.5 & 8.2 & 5.6 & 5.0 & $\underline{4.8}$ & 5.0 & 5.2 & 5.3 & 5.3 & 5.3 \\
\hline $\mathrm{W}, \%$ & $\underline{1.1}$ & 1.4 & 1.4 & 1.3 & 1.4 & 1.2 & 1.2 & 1.3 & 1.5 & 1.6 \\
\hline$e_{\mathrm{MFDE}}, \%$ & 4.4 & 4.8 & 5.4 & 6.0 & 6.3 & 6.5 & 6.6 & 7.5 & 8.0 & 8.4 \\
\hline $\mathrm{B}, \%$ & 10.1 & 11.1 & 12.5 & 14.1 & 16.6 & 15.6 & 16.8 & 19.2 & 21.8 & 23.8 \\
\hline $\mathrm{W}, \%$ & $\underline{3.0}$ & 3.1 & 3.5 & 3.8 & 3.6 & 4.0 & 4.0 & 4.4 & 4.3 & 4.4 \\
\hline \multicolumn{11}{|c|}{ Noise: $10 \%$ (actual: B 9.83\%; W 10.0\%); $\theta=0.222$} \\
\hline$\left(\xi_{i}, \eta_{i}\right)$ & $(1,0)$ & $(0,1)$ & $(1,-1)$ & $(1,1)$ & $(2,0)$ & $\overline{(0,2)}$ & $(0,5)$ & $(0,4)$ & $(2,1)$ & $(2,-1)$ \\
\hline Entropy & 1.0 & 2.4 & 3.2 & 3.7 & 3.7 & 3.0 & 1.9 & 1.1 & 0.7 & 0.5 \\
\hline$e_{\mathrm{DUDE}}, \%$ & 6.1 & 5.0 & 4.5 & 4.2 & 4.2 & 4.0 & 4.2 & 4.5 & 4.9 & 5.4 \\
\hline $\mathrm{B}, \%$ & 18.3 & 17.6 & 11.9 & 11.4 & 10.9 & 10.5 & 10.5 & 10.5 & 10.4 & $\underline{10.2}$ \\
\hline $\mathrm{W}, \%$ & 2.9 & 1.7 & 2.5 & 2.3 & 2.4 & 2.3 & 2.5 & 2.9 & 3.5 & 4.2 \\
\hline MFDE, $\%$ & 7.3 & 6.3 & 6.5 & 6.8 & 7.1 & 7.3 & 7.4 & 8.2 & 8.6 & 9.0 \\
\hline $\mathrm{B}, \%$ & 13.8 & $\underline{13.7}$ & 15.0 & 16.2 & 18.6 & 17.8 & 18.8 & 21.1 & 23.5 & 25.4 \\
\hline $\mathrm{W}, \%$ & 5.5 & 4.3 & 4.3 & 4.3 & $\underline{4.0}$ & 4.5 & 4.3 & 4.7 & 4.7 & 4.7 \\
\hline \multicolumn{11}{|c|}{ Noise: $20 \%$ (actual: B 19.8\%; W 19.9\%); $\theta=0.355$} \\
\hline$\left(\xi_{i}, \eta_{i}\right)$ & $(1,0)$ & $\overline{(0,1)}$ & $(1,-1)$ & $(1,1)$ & $(2,0)$ & $\overline{(0,2)}$ & $\overline{(0,5)}$ & $\overline{(0,4)}$ & $\overline{(2,1)}$ & $\overline{(2,-1)}$ \\
\hline Entropy & 1.0 & 2.3 & 3.2 & 3.4 & 3.5 & 3.5 & 2.2 & 1.2 & 0.7 & 0.5 \\
\hline$e_{\mathrm{DUDE}}, \%$ & 13.0 & 10.4 & 9.7 & 9.4 & 9.3 & 9.4 & 10.7 & 11.6 & 13.1 & 15.0 \\
\hline $\mathrm{B}, \%$ & 29.9 & 28.2 & 30.0 & 27.8 & 26.2 & 25.6 & 25.6 & 23.5 & 21.3 & $\underline{20.4}$ \\
\hline $\mathrm{W}, \%$ & 8.5 & 5.7 & $\underline{4.4}$ & 4.6 & 4.8 & 5.1 & 6.7 & 8.5 & 10.9 & 13.5 \\
\hline MFDE,$\%$ & 15.3 & 12.0 & 10.9 & 10.3 & 10.2 & 10.1 & 9.9 & 10.2 & 10.5 & 10.8 \\
\hline $\mathrm{B}, \%$ & 22.4 & $\underline{21.1}$ & 21.4 & 22.0 & 24.0 & 23.7 & 24.9 & 26.9 & 28.1 & 29.2 \\
\hline $\mathrm{W}, \%$ & 13.4 & 9.6 & 8.2 & 7.1 & 6.5 & 6.4 & 5.9 & $\underline{5.7}$ & 5.8 & 5.9 \\
\hline
\end{tabular}

the opposite way because the larger contexts result in more corrupted black areas (up to $64.5 \%$ ) while the white background becomes completely noiseless. For the larger noise level, the trends are even more transparent. With the small-size contexts $(K \leq 5)$, the overall quality of DUDE is better. But DUDE considerably corrupts the black area and simultaneously reduces the noise in the white area while MFDU less corrupts the former but less clean the latter. For larger contexts $(K>5)$, MFDU becomes more efficient. Anyway, as follows from Table 5, heuristic choice of the context gives slighly worse results than the maximum entropy based one.

For two other images with more balanced ratios of black and white areas, DUDE always outperforms MFDE under the adaptive context. But the difference tends to become low when the noise level increases. Once again, in "Cyrillic text" DUDE starts from an additional corruption of the smaller black area and cleans the white one to the larger extent. Then the corruption of the former decreases (but no lesser than the noise level) whereas the level of noise cleaning in the latter varies only slightly up and down. MFDU gradually corrupts the black area more and more while slightly improves the 
Table 4. Denoising of "Brodatz's Cane": 66\% black (B) and 34\% white (W) pixels (the same notation as in Table 2).

\begin{tabular}{|c|c|c|c|c|c|c|c|c|c|c|}
\hline Half-size $i$ & 1 & 2 & 3 & 4 & 5 & 6 & 7 & 8 & 9 & 10 \\
\hline \multicolumn{11}{|c|}{ Noise: 5\% (actual: B 4.98\%; W 4.99\%); $\theta=0.108$} \\
\hline$\left(\xi_{i}, \eta_{i}\right)$ & $(0,1)$ & $(1,0)$ & $(1,-1)$ & $(1,1)$ & $(0,2)$ & $\overline{(1,2)}$ & $(1,-2)$ & $(2,0)$ & $(2,-1)$ & $\overline{(2,1)}$ \\
\hline Entropy & 1.0 & 2.4 & 3.3 & 2.9 & 2.0 & 1.4 & 1.1 & 0.9 & 0.7 & 0.6 \\
\hline$e_{\mathrm{DUDE}}, \%$ & 2.2 & 1.8 & 1.6 & 1.4 & 1.5 & 1.7 & 2.0 & 2.2 & 2.5 & 2.7 \\
\hline $\mathrm{B}, \%$ & 1.7 & 1.6 & $\underline{1.0}$ & 1.1 & 1.2 & 1.4 & 1.6 & 1.8 & 2.1 & 2.2 \\
\hline $\mathrm{W}, \%$ & 3.1 & 2.1 & 2.7 & $\underline{2.0}$ & 2.1 & 2.2 & 2.6 & 2.9 & 3.2 & 3.4 \\
\hline$e_{\mathrm{MFDE}}, \%$ & 2.2 & 1.9 & 2.2 & 2.5 & 2.8 & 3.4 & 4.0 & 4.5 & 5.0 & 5.3 \\
\hline $\mathrm{B}, \%$ & 2.0 & 1.0 & 0.7 & 0.6 & 0.5 & 0.6 & 0.6 & 0.5 & 0.5 & $\underline{0.5}$ \\
\hline $\mathrm{W}, \%$ & 2.7 & 3.4 & 4.8 & 6.1 & 7.2 & 8.9 & 10.4 & 12.0 & 13.4 & 14.4 \\
\hline \multicolumn{11}{|c|}{ Noise: $10 \%$ (actual: B 9.76\%; W 9.79\%); $\theta=0.200$} \\
\hline$\left(\xi_{i}, \eta_{i}\right)$ & $(0,1)$ & $\overline{(1,1)}$ & $(1,-1)$ & $\overline{(1,1)}$ & $(0,2)$ & $(1,2)$ & $(2,0)$ & $(1,-2)$ & $(2,-1)$ & $(2,1)$ \\
\hline Entropy & 1.0 & 2.5 & 3.3 & 3.3 & 2.4 & 1.6 & 1.1 & 0.8 & 0.6 & 0.5 \\
\hline$e_{\mathrm{DUDE}}, \%$ & 4.9 & 3.5 & 3.2 & 2.7 & 2.8 & 3.5 & 4.1 & 5.0 & 5.8 & 6.5 \\
\hline $\mathrm{B}, \%$ & 4.2 & 2.2 & 1.9 & $\underline{1.6}$ & 1.8 & 2.5 & 3.1 & 4.1 & 5.0 & 5.9 \\
\hline $\mathrm{W}, \%$ & 6.3 & 6.1 & 5.7 & 4.7 & $\underline{4.5}$ & 5.3 & 5.8 & 6.6 & 7.2 & 7.5 \\
\hline$e_{\mathrm{MFDE}}, \%$ & 4.9 & 3.5 & 3.4 & 3.5 & 3.9 & 4.4 & 4.8 & 5.1 & 5.6 & 5.9 \\
\hline $\mathrm{B}, \%$ & 4.6 & 2.4 & 1.7 & 1.6 & 1.3 & 1.2 & 1.2 & 1.0 & 1.1 & $\underline{1.0}$ \\
\hline $\mathrm{W}, \%$ & 5.6 & $\underline{5.5}$ & 6.6 & 7.3 & 8.8 & 10.4 & 11.6 & 12.9 & 14.2 & 15.3 \\
\hline \multicolumn{11}{|c|}{ Noise: $20 \%$ (actual: B 19.7\%; W 20.1\%); $\theta=0.317$} \\
\hline$\left(\xi_{i}, \eta_{i}\right)$ & $(0,1)$ & $(1.0)$ & $(1,-1)$ & $\overline{(1,1)}$ & $\overline{(0,2)}$ & $\overline{(1,2)}$ & $\overline{(1,-2)}$ & $(2,0)$ & $\overline{(2,-1)}$ & $\overline{(2,1)}$ \\
\hline Entropy & 1.0 & 2.4 & 3.2 & 3.5 & 3.1 & 1.9 & 1.1 & 0.7 & 0.4 & 0.3 \\
\hline$e_{\mathrm{DUDE}}, \%$ & 13.2 & 9.6 & 8.6 & 7.6 & 8.0 & 9.7 & 12.1 & 14.8 & 16.8 & 18.3 \\
\hline $\mathrm{B}, \%$ & 11.8 & 7.7 & 5.0 & $\underline{3.9}$ & 5.0 & 6.7 & 9.6 & 13.1 & 15.8 & 17.7 \\
\hline $\mathrm{W}, \%$ & 15.5 & 13.0 & 15.3 & 14.5 & $\underline{13.7}$ & 15.1 & 16.6 & 17.8 & 18.3 & 19.1 \\
\hline$e_{\mathrm{MFDE}}, \%$ & 13.2 & 9.6 & 8.1 & 7.1 & 6.7 & 6.7 & 6.9 & 6.9 & 7.2 & 7.5 \\
\hline $\mathrm{B}, \%$ & 12.4 & 8.3 & 6.0 & 5.0 & 3.8 & 3.5 & 3.2 & 2.7 & 2.6 & $\underline{2.4}$ \\
\hline $\mathrm{W}, \%$ & 14.2 & 11.9 & 11.9 & $\underline{11.0}$ & 12.0 & 12.9 & 13.9 & 14.9 & 15.9 & $\overline{17.3}$ \\
\hline
\end{tabular}

Table 5. Denoising of "Stars": the nearest symmetric 8-neighbourhood.

\begin{tabular}{|lc|ccc|ccc|}
\hline \multirow{2}{*}{ Noise } & $\theta$ & \multicolumn{5}{|c|}{ Residual noise } \\
\cline { 3 - 8 } & & \multicolumn{3}{|c|}{$\begin{array}{c}\text { DUDE } \\
\text { DUDE } \%\end{array}$} & B-noise, \% & W-noise, $\%$ & \multicolumn{3}{c|}{ MFDE } \\
& & $e_{\text {MFDE }} \%$ & B-noise, \% & W-noise, \% \\
\hline $5 \%$ & 0.096 & 0.08 & 3.28 & 0.07 & 0.08 & 23.0 & 0.01 \\
$10 \%$ & 0.178 & 0.26 & 29.0 & 0.18 & 0.21 & 29.0 & 0.12 \\
$20 \%$ & 0.362 & 0.43 & 73.8 & 0.22 & 2.08 & 35.5 & 1.98 \\
\hline
\end{tabular}

noise removal from the white area when the context size is growing up. If the sizes of the black and white areas are closer ("Brodatz's Cane"), these differences as well as the differences in the total quality of denoising decrease, and at lower noise levels DUDE outperforms MFDE while for the higher noise MFDE turns to be slightly better. Both the denoisers in this case reduce about $60-70 \%$ of the initial noise.

Comparisons to a heuristic context selection show that in the most cases the adaptive choice returns better contexts. It is worthy to mention that the best contexts are 
not necessarily continuous. For "Stars" and "Cyrillic text", the window size to compare possible pixel pairs almost does not impact the choice of the very first characteristic neighbours. The characteristic context for the periodic texture "Brodatz's Cane" estimated in the smaller window $13 \times 13$ differs from the context in the larger window $81 \times 81$ (see Fig. 4). It should be noted that under the manually selected best threshold $\theta$, the latter disjoint context results in even slightly better denoising. But when the thresolds are estimated from the noisy image itself, the contexts for the smaller windows give better results.

\section{Conclusions}

These and other similar experiments show that, in principle, the contexts for DUDE may be chosen using the maximum likelihood structure of pairwise signal dependences in noisy binary images. If the noiseless black and white area are not considerably unbalanced, the active DUDE with the adaptively chosen context mostly outperforms the more conventional passive MFDE. But the higher the noise, the smaller the difference between both the denoisers. The size of the context can roughly be related to the maximum entropy of the distribution of the minimum relative frequencies of signals with the same contexts (these frequencies are used for DUDE itself, too).

When denoising an image, MFDE always additionally corrupts the non-dominant area in favour of cleaning the dominant one. DUDE is also biased to the latter area but corrupts it to the lesser extent.

\section{References}

1. M. Berman: Automated smoothing of image and other regularly spaced data. IEEE Trans. Pattern Anal. Machine Intell., vol. 16, 1994, 460-648.

2. A. Blake and A. Zisserman: Visual Reconstruction. MIT Press: Cambridge, MA, 1987.

3. S. Geman and D. Geman, Stochastic relaxation, Gibbs distribution, and Bayesian restoration of images. IEEE Trans. Pattern Anal. Machine Intell., vol. 6, 1984, 721-741.

4. G. L. Gimel'farb: Image Textures and Gibbs Random Fields. Dordrecht: Kluwer Academic, 1999.

5. L. Rudin, S. Osher, and E. Fatemi: Nonlinear total variation based noise removal algorithm. Physica. vol. 60D, 1992, 259-268.

6. T. Weissman, E. Ordentlich, G. Seroussi, S. Verdu, and M. Weinberger: Universal Discrete Denoising: Known Channel. Technical Report HPL-2003-29. IT Research, HP Labs Palo Alto, February 10, 2003. 